\title{
Two-component Gaussian core model: Strong-coupling limit, Bjerrum pairs, and gas-liquid phase transition
}

Cite as: J. Chem. Phys. 148, 024904 (2018); https://doi.org/10.1063/1.5006947

Submitted: 28 September 2017 . Accepted: 27 December 2017 . Published Online: 11 January 2018

Derek Frydel (D), and Yan Levin (D)
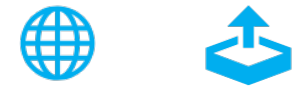

View Online

Export Citation

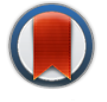

\section{ARTICLES YOU MAY BE INTERESTED IN}

\section{Perspective: Dissipative particle dynamics}

The Journal of Chemical Physics 146, 150901 (2017); https://doi.org/10.1063/1.4979514

On the mechanical stability of the body-centered cubic phase and the emergence of a metastable cl16 phase in classical hard sphere solids

The Journal of Chemical Physics 148, 024502 (2018); https://doi.org/10.1063/1.5009099

Bjerrum pairs in ionic solutions: A Poisson-Boltzmann approach

The Journal of Chemical Physics 146, 194904 (2017); https://doi.org/10.1063/1.4982885

Where in the world is AIP Publishing? Find out where we are exhibiting next 


\title{
Two-component Gaussian core model: Strong-coupling limit, Bjerrum pairs, and gas-liquid phase transition
}

\author{
Derek Frydel ${ }^{1,2, a)}$ and Yan Levin ${ }^{2}$ \\ ${ }^{1}$ Federico Santa María Technical University, Avda. Vicuña Mackenna 3939, San Joaquín, Santiago, Chile \\ ${ }^{2}$ Institute of Physics, The Federal University of Rio Grande do Sul, Porto Alegre 91501-970, Brazil
}

(Received 28 September 2017; accepted 27 December 2017; published online 11 January 2018)

\begin{abstract}
In the present work, we investigate a gas-liquid transition in a two-component Gaussian core model, where particles of the same species repel and those of different species attract. Unlike a similar transition in a one-component system with particles having attractive interactions at long separations and repulsive interactions at short separations, a transition in the two-component system is not driven solely by interactions but by a specific feature of the interactions, the correlations. This leads to extremely low critical temperature, as correlations are dominant in the strong-coupling limit. By carrying out various approximations based on standard liquid-state methods, we show that a gasliquid transition of the two-component system poses a challenging theoretical problem. Published by AIP Publishing. https://doi.org/10.1063/1.5006947
\end{abstract}

\section{INTRODUCTION}

A two-component fluid with interactions

$$
u_{i j}(r)=\left\{\begin{array}{cc}
u(r), & \text { if } i=j, \\
-u(r), & \text { if } i \neq j,
\end{array}\right.
$$

where the indices designate the two components, is best exemplified by electrolytes. Both in experiments and idealized representations, such as primitive models ${ }^{1-5}$ or penetrable ions, ${ }^{6-13}$ electrolytes undergo a gas-liquid transition. A similar transition is expected for any two-component fluid as long as interactions obey Eq. (1), and it can be understood by adopting an effective one-component description, where particles of one species experience mediated attractive interactions due to averaged contributions of the second species. The attractions eventually lead to phase transition.

A theoretical challenge posed by the models conforming to Eq. (1) is that they can only be described by a theoretical framework containing correlations, ${ }^{14}$ and therefore, a straightforward application of mean-field techniques is of no use. The simplest theory of correlations is the random phase approximation (RPA), which is equivalent to a one-loop expansion around the mean-field solution (or the saddle-point). ${ }^{15,16}$ Corrections due to the strong-coupling limit effects can be incorporated into the RPA framework by an explicit incorporation of Bjerrum pairs, which are dimers formed between particles of opposite species. ${ }^{1,2,6,17,18}$ For the primitive model, this procedure correctly shifts the critical point of a gasliquid transition to higher densities. On the other hand, for penetrable ions, the same procedure leads to no satisfactory results. ${ }^{6}$

Motivated by this theoretical difficulty of treating fluids with the binary interactions of the form presented in Eq. (1),

a)dfrydel@gmail.com the present article considers a fluid of Gaussian particles, the so-called Gaussian core model (GCM). Like penetrable ions, Gaussian particles are penetrable, but unlike penetrable ions, Gaussian interactions are short-ranged. A one-component GCM fluid has been studied extensively in the past by numerous groups. ${ }^{20-26}$ The two-component version of the GCM model, but for repulsive-only interactions, was investigated in Ref. 27. The two-component GCM fluid with interactions satisfying Eq. (1) was briefly introduced in Ref. 15 as a testing ground for the generalized-RPA approximation.

This work is organized as follows. In Sec. II, we introduce the GCM model. In Sec. III, we present the simulation results, focusing on the location of the critical point and the structure of a fluid with special view to dimer formation. In Sec. IV, we analyze the GCM model using a number of approximations. Finally, in Sec. V, we conclude the work.

\section{THE GAUSSIAN CORE MODEL}

Particles in the GCM model interact via the Gaussian potential, $u(r)=\epsilon e^{-r^{2} / \sigma^{2}}$, and for the two-component system considered in the present work, the interactions are

$$
u_{i j}(r)=\left\{\begin{array}{cc}
\varepsilon e^{-r^{2} / \sigma^{2}}, & \text { if } i=j, \\
-\varepsilon e^{-r^{2} / \sigma^{2}}, & \text { if } i \neq j,
\end{array}\right.
$$

where the indices $i, j=1,2$ designate different species, $\varepsilon$ is the depth of the potential, and $\sigma$ is the particle diameter. In the following, we use the physical quantities reduced by $\sigma, \varepsilon$, and the Boltzmann constant $k_{B}$. The reduced length is $r^{*}=r / \sigma$, and the reduced density is $\rho^{*}=\rho \sigma^{3}$. Then, the reduced temperature is $T^{*}=k_{B} T / \varepsilon$, the reduced pressure is $P^{*}=P \sigma^{3} /\left(k_{B} T\right)$, and the strength of the Gaussian potential in relation to thermal energy is $\varepsilon^{*}=1 / T^{*}=\varepsilon /\left(k_{B} T\right)$.

Simulation results in the present work are from the standard canonical Monte Carlo (MC) simulations $(N, V, T)$. Because the pair potentials of the GCM fluid are bounded, 
there is no constraint for the displacement length of attempted moves, which could be very long. In our simulations, we set this length to ensure that the acceptance ratio is larger than $50 \%$ and less than $80 \%$. All simulations are performed in a cubic box with periodic boundary conditions in all three directions. The total number of particles in the box is $N_{1}+N_{2}=1000$, and the bulk densities $\rho_{1}$ and $\rho_{2}$ are controlled by the box size L. As $N_{1}=N_{2}=N / 2$, the density of each species is $\rho_{1}=\rho_{2}$ $=\rho / 2$.

\section{SIMULATION RESULTS}

\section{A. Pressure isotherms and the critical point}

The presence and location of the critical point of a gas-liquid phase transition is determined from pressure isotherms, where we know that the critical temperature isotherm includes a stationary inflection point, $\left(\frac{\partial P}{\partial \rho}\right)_{T}$ $=\left(\frac{\partial^{2} P}{\partial \rho^{2}}\right)_{T}=0$. Figure 1 displays a number of isotherms generated by simulations. A gradual emergence of a plateau in the shape of an isotherm with decreasing temperature indicates the approaching critical temperature. The critical point is estimated to be roughly at $T_{c}^{*} \approx 0.03$ and $\rho_{c}^{*} \approx 0.6$.

Such a low critical temperature is a signature of a phase transition that is driven by correlations. Other systems with the binary interactions of the form presented in Eq. (1) exhibit a similar behavior. For example, the critical temperature of penetrable ions is $T_{c}^{*} \approx 0.02^{8}$ and that of the restrictive primitive model is $T_{c}^{*} \approx 0.07 .{ }^{1}$ By contrast, the critical temperature of an analogous gas-liquid phase transition of a one-component Lennard-Jones fluid is $T_{c}^{*} \approx 1.1,{ }^{19}$ which roughly corresponds to an equivalence between the thermal energy and the potential energy at minimum.

We note that normally a critical point depends on a size of a simulation box $L .{ }^{11}$ For the critical temperature, this dependence is expressed as $T_{c}(L)=T_{c}+L^{-a}$, where $T_{c}$ is the critical temperature in the thermodynamic limit, and the value of $a$ for the Ising model is $\sim 2.44$. In the present work, we are interested in general properties of a fluid prior to the onset of a phase transition, and a rough estimate of the critical point is sufficient for our purposes. To check finite size effects, we generate a number of isotherms for $N=2000$ particles, but we find no change in the results.

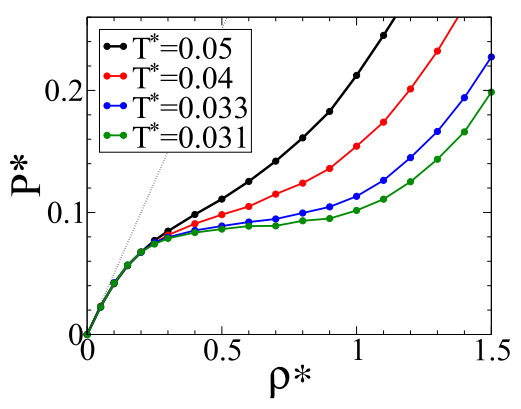

FIG. 1. Pressure isotherms for the two-component GCM fluid generated by MC simulations. The dashed line designates an ideal-gas pressure for $N / 2$ dimers, $P^{*}=\rho^{*} / 2$.

\section{B. Correlation functions}

A unique feature of a two-component system with the binary interactions of the form presented in Eq. (1) is the formation of dimers between particles of opposite species, known as Bjerrum pairs in the context of electrolytes. At low temperature and density, these pairs dominate the fluid structure and, because of their stability, can be regarded as a third component. It is not clear, however, what effect, if any, the pairs may have on a phase-transition and whether a successful theory of a phase-transition is required to incorporate pair formation.

To examine this question, in this section, we consider a number of correlation functions. The two relevant correlations are as follows: correlations between particles of the same species, $h_{11}(r)$, and correlations between particles of different species, $h_{12}(r)$. In Fig. 2, we plot these functions for different densities slightly above the critical temperature, $T^{*} \gtrsim T_{c}^{*} \approx 0.03$.

First, we consider the function $h_{12}(r)$. For the lowest density, the correlation function is dominated by a sharp peak at $r=0$ and the absence of oscillations, indicating the absence of the secondary structures. This suggests that the particles exist as pairs and the system can be regarded as an ideal-gas of $N / 2$ dimers. This is confirmed by an incipient agreement in Fig. 1 between the pressure isotherms generated by simulations and the linear behavior $P^{*}=\rho^{*} / 2$. Dimers disintegrate with increasing density, which is seen as $h_{12}(r)$ develops a usual oscillatory structure.

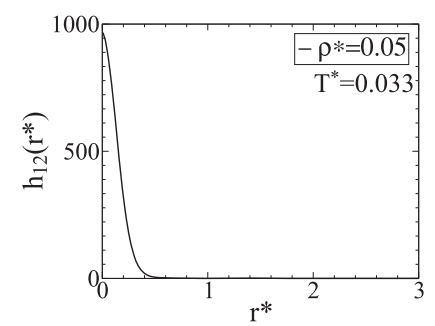

(a)

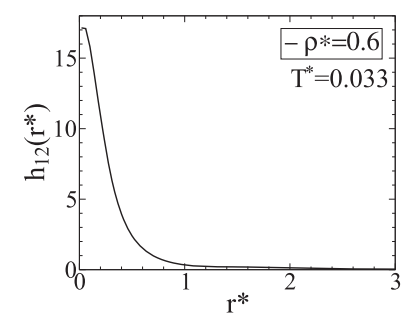

(c)

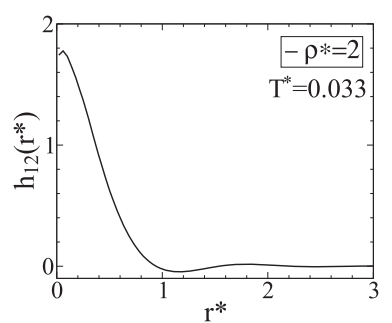

(e)

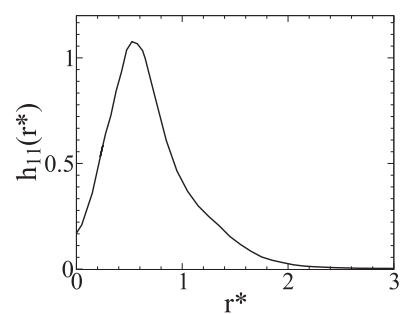

(b)

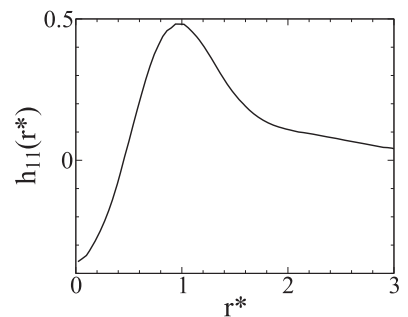

(d)

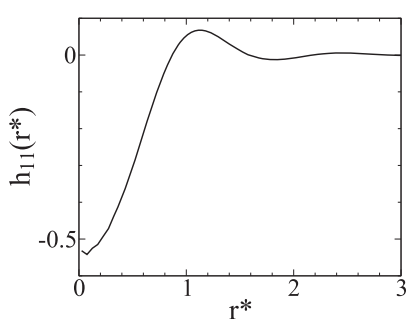

(f)
FIG. 2. Pair correlation functions, $h_{11}(r)$ and $h_{12}(r)$, for the two-component GCM fluid for $T^{*}=0.033 \gtrsim T_{c}^{*}$ for three densities $\rho^{*}=0.05,0.6$, and 2 . 
We examine next the function $h_{11}(r)$. Because the bare interactions between particles of the same species are repulsive, $h_{11}(r)$ is expected to feature a correlation hole, a region of negative correlations around $r=0$. This expectation is satisfied for densities $\rho^{*}=0.6$ and $\rho^{*}=2$. But for $\rho^{*}=0.05$, the correlation hole vanishes and is substituted by a region of positive correlations, indicating effective attractions. Since from $h_{12}(r)$ we know that dimers dominate a fluid structure at $\rho^{*}=0.05$, the effective attractions imply attractions between dimers. This, in turn, explains a gradual deviation of pressure isotherms from the ideal-gas behavior $P^{*}=\rho^{*} / 2$, suggesting a corrected low density ansatz $P^{*}=\rho^{*} / 2+B_{2} \rho^{* 2}$.

The above discussion suggests that it may be interesting to adopt an effective one-component description by integrating out the degrees of freedom of the second component, analogous to the concept of depletion interactions. ${ }^{28-30}$ At low density, effective interactions can be obtained by inverting a pair correlation function, $\beta u_{\text {eff }}=-\log h_{11}(r)$, shown in Fig. 3 for $\rho^{*}=0.05$ and $T^{*}=0.033$. The resulting effective potential is everywhere negative with a minimum at $r^{*} \approx 0.5$. We attempt next to carry out a simulation for a one-component system for $\rho^{*}=0.025$ and interactions $u_{\mathrm{eff}}(r)$. We find this system thermodynamically unstable, with all particles collapsing into a single cluster with infinite overlaps. To make the description more realistic and prevent such a collapse, one would need to include three-body and perhaps higherbody effective interactions. This, however, greatly complicates the required computations and, in effect, makes the effective one-component approach unfeasible.

\section{Pairs}

Another way of looking at a fluid structure, and, especially, formation of dimers, is by analyzing the quantity

$$
C_{i j}=\rho_{i} \int_{0}^{\infty} d r 4 \pi r^{2} h_{i j}(r)
$$

where $\rho_{i} h_{i j}(r)$ is a density perturbation of a species " $i$ " around a fixed fluid particle of a species " $j$ " (we recall that $\rho_{i}=\rho / 2$ ).

The data points obtained from simulations for a low density $\rho^{*}=0.05$ are plotted in Fig. 4 as a function of $\varepsilon^{*}=1 / T^{*}$, prior to the onset of a phase transition. To better understand the quantity $C_{12}$ in Fig. 4(a), it is helpful to keep in mind the shape of a correlation function $h_{12}(r)$ for the same density in Fig. 2(a). The emergence of quasi-stable pairs corresponds to $C_{12} \approx 1$. Initially $C_{12}$ increases linearly, and at $\varepsilon^{*}=10$ (or

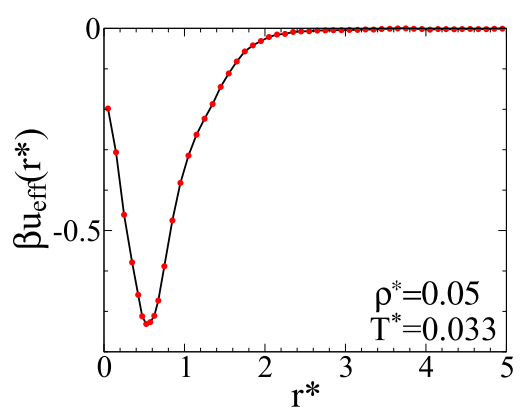

FIG. 3. Mediated interactions between particles of the same species defined as $\beta u_{\mathrm{eff}}(r)=-\log h_{11}(r)$, for density $\rho^{*}=0.05$ and temperature $T^{*}=0.033 \gtrsim T_{c}^{*}$.

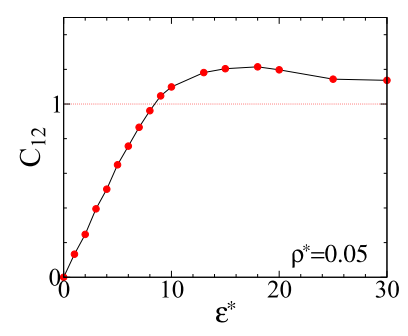

(a)

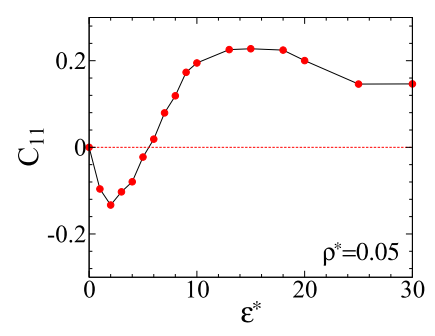

(b)
FIG. 4. The quantity $C_{i j}=\rho_{i} \int_{0}^{\infty} d r 4 \pi r^{2} h_{i j}(r)$ as a function of $\varepsilon^{*}=1 / T^{*}$ for $\rho^{*}=0.05$, prior to the onset of phase transition. The data points are from simulations.

$T^{*}=0.1$ ), all particles are paired. However, instead of saturating at $1, C_{12}$ continues to increase as a consequence of mediated attractive interactions, indicating that pairs are not ideal-gas particles.

Next, we interpret the data points for $C_{11}$ in Fig. 4(b). In the case of weak interactions, $C_{11}$ is negative, reflecting the presence of a correlation hole in $h_{11}(r)$. These negative correlations begin to disappear around $\varepsilon^{*} \approx 2$ and then at $\varepsilon^{*} \approx 6$, the quantity $C_{11}$ changes sign and becomes positive, indicating the onset of effective attractive interactions.

In analogy to Coulomb particles, we consider next the quantity

$$
C_{d}=\rho_{i} \int_{0}^{\infty} d r 4 \pi r^{2}\left[h_{i i}(r)-h_{i j}(r)\right] \geq-1,
$$

where $C_{d}$ designates a "charge" that a fixed fluid particle attracts. By contrast, for a Coulomb system $C_{d}=-1$, as a consequence of long-range interactions, and implies the perfect screening. This exact condition is referred to as the (zero-order) Stillinger-Lovett sum rule. ${ }^{31-33}$ On the other hand, the GCM two-component fluid achieves perfect screening gradually as $T^{*} \rightarrow 0$ or $\rho^{*} \rightarrow \infty$.

The plots of Fig. 5 are fitted to simple functional forms. A fast exponential decay in Fig. 5(a) agrees with the notion that dimers dominate the fluid structure at low densities for $T^{*}<0.1 . C_{d}$ as a function of $\rho^{*}$ and for $T^{*}=1$ in Fig. 5(b), on the other hand, can be fit to an algebraic decay obtained from the RPA, which is a weak-coupling theory, and does not capture the formation of dimers.

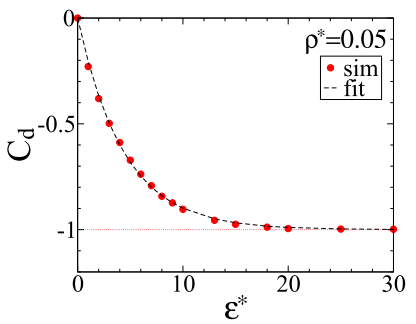

(a)

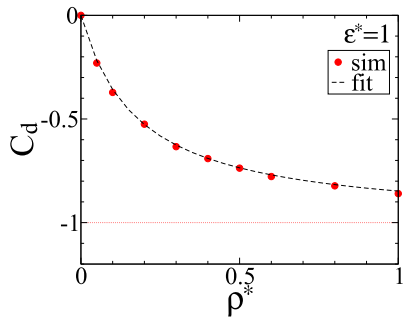

(b)
FIG. 5. The quantity $C_{d}=\rho_{i} \int_{0}^{\infty} d r 4 \pi r^{2}\left\{h_{i i}(r)-h_{i j}(r)\right\}$, designating the total "charge" a fixed particle attracts, (a) as a function of $\varepsilon^{*}$ for $\rho^{*}=0.05$ and (b) as a function of $\rho^{*}$ for $\varepsilon^{*}=1 . C_{d}$ is bounded from below by -1 . The fits to the data points for respective plots are $C_{d}=e^{-\varepsilon^{*} / \varepsilon_{0}^{*}}-1$, with $\varepsilon_{0}^{*} \approx 4.3$, and $C_{d}=\frac{\pi^{3 / 2} \varepsilon^{*} \rho^{*}}{1+\pi^{3 / 2} \varepsilon^{*} \rho^{*}}$ (obtained from the RPA). 


\section{THEORETICAL ANALYSIS}

\section{A. Random phase approximation}

The free energy density for the two-component system with interactions in Eq. (1) has two main contributions,

$$
f=f_{\text {id }}+f_{c},
$$

where the ideal-gas contribution is

$$
f_{\text {id }}=k_{B} T \rho\left(\log \frac{\rho \Lambda^{3}}{2}-1\right)
$$

( $\Lambda$ is the de Broglie wavelength) and the expression for the correlation free energy density can be obtained from the adiabatic connection, ${ }^{15,16}$ wherein the pair interaction $\lambda u(r)$ is gradually turned on by changing $\lambda$ from $0 \rightarrow 1$. The resulting expression for the present two-component homogeneous system is

$$
f_{c}=\pi \rho^{2} \int_{0}^{\infty} d r r^{2} u(r) \int_{0}^{1} d \lambda h_{d}^{\lambda}(r)
$$

where $h_{d}^{\lambda}(r)=h_{11}^{\lambda}(r)-h_{12}^{\lambda}(r)$ and the superscript $\lambda$ indicates the correlation function for particles with interactions $\lambda u(r)$.

The correlation function in Eq. (7) is obtained from the Ornstein-Zernike (OZ) equation,

$$
h_{i j}^{\lambda}(r)=-\beta c_{i j}^{\lambda}(r)-\beta \sum_{k=1}^{2} \rho_{k} \int d \mathbf{r}^{\prime} h_{k j}^{\lambda}\left(r^{\prime}\right) c_{i k}^{\lambda}\left(\left|\mathbf{r}^{\prime}-\mathbf{r}\right|\right),
$$

where within the RPA the direct correlation function is approximated as $c_{i j}^{\lambda, \text { rpa }}(r)=-\lambda \beta u_{i j}(r)$. This in turn implies that $h_{11}^{\lambda, \text { rpa }}(r)=-h_{12}^{\lambda, \text { rpa }}(r)=h_{\lambda}^{\text {rpa }}(r)$, where the function $h_{\lambda}^{\text {rpa }}(r)$ is obtained from the modified OZ equation,

$$
h_{\lambda}^{\mathrm{rpa}}(r)+\beta \lambda u(r)=-\beta \lambda \rho \int d \mathbf{r}^{\prime} h_{\lambda}^{\mathrm{rpa}}\left(r^{\prime}\right) u\left(\left|\mathbf{r}^{\prime}-\mathbf{r}\right|\right),
$$

leading to the approximate correlation free energy,

$$
\beta f_{\mathrm{c}}^{\mathrm{rpa}}=-\frac{\rho}{2} \int_{0}^{1} d \lambda \frac{h_{\lambda}^{\mathrm{rpa}}(0)+\lambda \beta u(0)}{\lambda} .
$$

To obtain analytical results, Eq. (9) is Fourier transformed,

$$
\hat{h}_{\lambda}^{\mathrm{raa}}(k)=-\frac{\beta \lambda \hat{u}(k)}{1+\beta \lambda \rho \hat{u}(k)} .
$$

After the Fourier inversion, the correlation free energy becomes

$$
\beta f_{\mathrm{c}}^{\mathrm{rpa}}=\frac{1}{4 \pi^{2}} \int_{0}^{\infty} d k k^{2}(\log [1+\rho \beta \hat{u}(k)]-\rho \beta \hat{u}(k)),
$$

which after substitution $\hat{u}(k)=\sigma^{3} \pi^{3 / 2} e^{-k^{2} \sigma^{2} / 4}$ evaluates to

$$
\beta f_{\mathrm{c}}^{\mathrm{rpa}}=-\frac{\varepsilon^{*} \rho}{2}\left\{1+\frac{\mathrm{Li}_{5 / 2}\left[-\varepsilon^{*} \eta\right]}{\varepsilon^{*} \eta}\right\}
$$

where $\eta=(\sigma \sqrt{\pi})^{3} \rho$, and $\operatorname{Li}_{m}(x)=\sum_{n=1}^{\infty} \frac{x^{n}}{n^{m}}$ is a polylogarithm.

To locate the critical point, we study pressure isotherms. The expression of pressure is obtained from either the

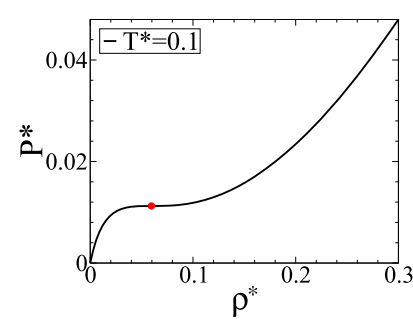

(a)

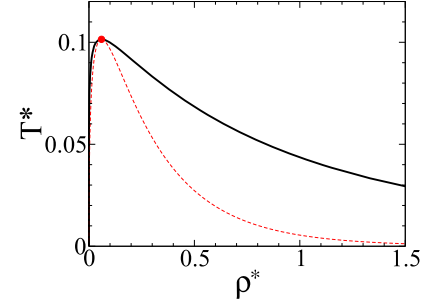

(b)
FIG. 6. (a) Pressure isotherm at a critical temperature, $T_{c}^{*} \approx 0.1$, from the RPA. The red point at $\rho_{c}^{*} \approx 0.06$ designates the critical density. (b) Phase diagram within the RPA. The spinodal lines are indicated with the dashed line, and the coexistence region is indicated with the solid line.

thermodynamic definition $P=-\frac{\partial F}{\partial V}=\rho \frac{\partial f}{\partial \rho}-f$ or the virial equation

$$
\frac{\beta P}{\rho}=1-\frac{\pi \rho}{3} \int_{0}^{\infty} d r r^{3} \frac{\partial \beta u(r)}{\partial r} h_{d}(r),
$$

where, within the RPA, $h_{d}^{\mathrm{rpa}}(r)=2 h^{\mathrm{rpa}}(r)$ and $h^{\mathrm{rpa}}(r)$ satisfies Eq. (9) for $\lambda=1$. The resulting formula is

$$
\frac{\beta P^{\mathrm{rpa}}}{\rho}=1+\frac{\varepsilon^{*}}{2}\left[\frac{\mathrm{Li}_{5 / 2}\left(-\varepsilon^{*} \eta\right)-\mathrm{Li}_{3 / 2}\left(-\varepsilon^{*} \eta\right)}{\varepsilon^{*} \eta}\right]
$$

The critical point is found at $T_{c}^{*} \approx 0.1$ (or $\varepsilon_{c}^{*} \approx 9.85$ ) and $\rho_{c}^{*} \approx 0.06$; see Fig. 6(a). The entire phase diagram of the coexistence region is shown in Fig. 6(b). The spinodal lines (designating a metastable region) correspond to the local condition $\frac{\partial^{2} f}{\partial \rho^{2}}=0$, while the coexistence region is constructed by the global consideration of the free energy, using the Maxwell construction.

The RPA critical temperature is considerably higher than that obtained from simulations, while the critical density is considerably lower. To understand some of the causes of this disparity, we look into the fluid structure and the behavior of pairs. The quantity $C_{d}$ defined in Eq. (4) within the RPA is

$$
C_{d}^{\mathrm{rpa}}=\rho \hat{h}^{\mathrm{rpa}}(0)=-\frac{\varepsilon^{*} \eta}{1+\varepsilon^{*} \eta},
$$

where we recall that $\eta=(\sigma \sqrt{\pi})^{3} \rho$. As this algebraic behavior agrees with the high temperature results in Fig. 5(b), it fails to agree at low temperatures, or the strong-coupling limit, and low density in Fig. 5(a), where an exponential decay of $C_{d}$ to -1 indicates the formation of dimers (Fig. 7).

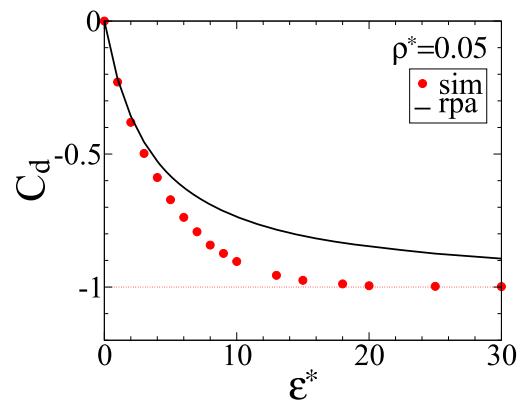

FIG. 7. The same as in Fig. 5 but now along with the RPA prediction given in Eq. (16). 
The absence of dimers within the RPA can, furthermore, be attested by the structure of correlations shown in Fig. 8 for $T_{c}^{*} \approx 0.1$. First, the correlations always exhibit oscillatory structure. Second, since within the RPA $h_{11}(r)=-h_{12}(r)$, the function $h_{11}(r)$ always exhibits a correlation hole so that the mediated attractive interactions between particles of the same species never arise. This is seen by examining the quantity $C_{11}$, within the RPA given by

$$
C_{11}^{\mathrm{rpa}}=-\frac{1}{2} \frac{\varepsilon^{*} \eta}{1+\varepsilon^{*} \eta},
$$

which is dominated by negative correlations for any set of parameters.

\section{RPA in general dimensions}

In this section, we briefly consider the dimensiondependence of the critical point. As seen from the simulation results in Fig. 9, the critical temperature and density decrease with reduced dimensionality, as $d=4 \rightarrow 3 \rightarrow 2$, and in $d=1$, there is no phase transition.

The Fourier transformed Gaussian pair potential for a general dimension is $\beta \hat{u}(k)=(\sigma \sqrt{\pi})^{d} \varepsilon e^{-k^{2} \sigma^{2} / 4}$, and the correlational free energy becomes

$$
\begin{aligned}
\beta f_{\mathrm{c}}^{\mathrm{rpa}}= & \frac{1}{(4 \pi)^{d / 2} \Gamma(d / 2)} \\
& \times \int_{0}^{\infty} d k k^{d-1}(\log [1+\rho \beta \hat{u}(k)]-\rho \beta \hat{u}(k)),
\end{aligned}
$$

which evaluates to

$$
\beta f_{\mathrm{c}}^{\mathrm{rpa}}=-\frac{\varepsilon \rho}{2}\left\{1+\frac{\mathrm{Li}_{d / 2+1}[-\varepsilon \eta]}{\varepsilon \eta}\right\},
$$

where $\eta=(\sigma \sqrt{\pi})^{d} \rho$. Using the thermodynamic definition, $\beta P=\rho+\rho \frac{\partial \beta f_{\mathrm{c}}}{\partial \rho}-\beta f_{\mathrm{c}}$, and the pressure is given as

$$
\beta P=\rho+\frac{\varepsilon \rho}{2}\left\{\frac{\operatorname{Li}_{d / 2+1}(-\varepsilon \eta)-\mathrm{Li}_{d / 2}(-\varepsilon \eta)}{\varepsilon \eta}\right\} .
$$

Critical temperatures from pressure isotherms are plotted in Fig. 10 as a function of $d$, together with the data points obtained from simulations. We discover that the RPA fails even in capturing a general trend: as dimensionality increases, the RPA critical temperature decreases. This shows general inadequacy of the RPA for describing the strong-coupling limit of two-component systems with interactions of the form

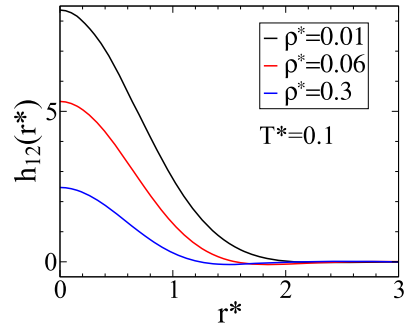

(a)

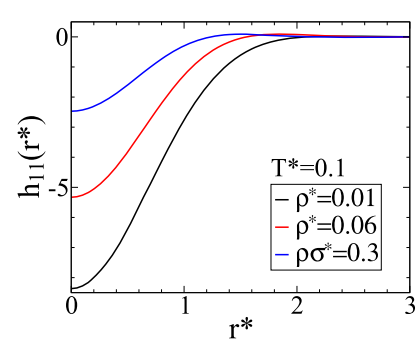

(b)
FIG. 8. Pair correlation functions predicted by the RPA slightly above the critical temperature at $T_{c}^{*} \approx 0.1$ for densities $\rho^{*}=0.01,0.06$, and 0.3 .

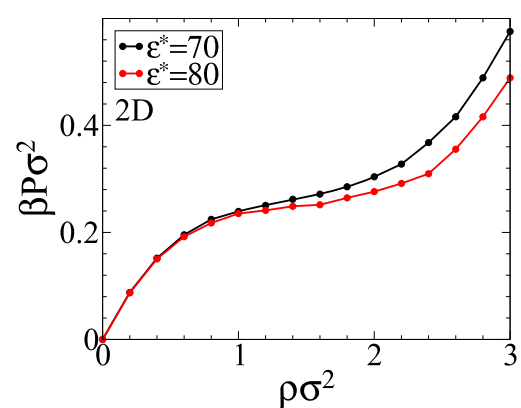

(a)

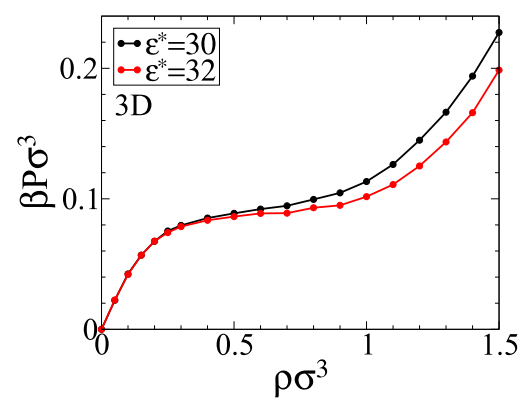

(b)

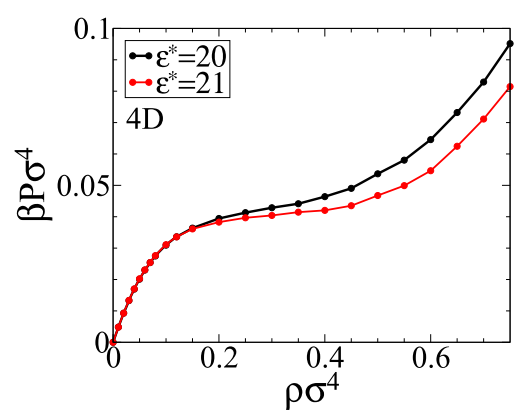

(c)

FIG. 9. Pressure isotherms for the two-component GCM fluid in various dimensions, $d=2,3,4$, slightly above the critical temperature. The data points are from the MC simulation for $N=1000$ particles.

presented in Eq. (1). In Secs. IV B and IV C, we consider other possible approaches.

\section{B. Correlation functions from the mean-field approximation}

In the next attempt to treat theoretically the present system, we define a correlation function in terms of a density

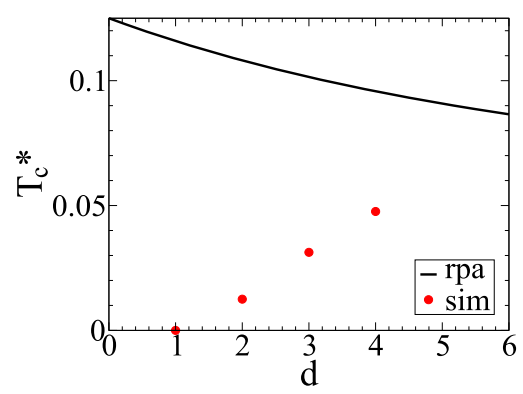

FIG. 10. Critical temperature as a function of dimensionality $d$ for the RPA compared with the data points from MC simulations. 
perturbation. A fixed fluid particle of the type " 1 " at the coordinate origin generates density perturbations that, within the mean-field approximation, are given by

$$
\begin{gathered}
\rho_{1}(r)=\frac{\rho}{2} e^{-\beta u(r)} e^{-\beta w(r)}, \\
\rho_{2}(r)=\frac{\rho}{2} e^{\beta u(r)} e^{\beta w(r)},
\end{gathered}
$$

where

$$
w(r)=\int d \mathbf{r}^{\prime} u\left(\mathbf{r}, \mathbf{r}^{\prime}\right)\left[\rho_{1}\left(r^{\prime}\right)-\rho_{2}\left(r^{\prime}\right)\right]
$$

is the mean-potential due to an average distribution of all particles in the system. Using the formal definitions $\rho_{1}(r)$ $=\frac{\rho}{2}\left[h_{11}(r)+1\right]$ and $\rho_{2}(r)=\frac{\rho}{2}\left[h_{12}(r)+1\right]$, the above equations transform into

$$
\begin{array}{r}
h_{11}(r)=e^{-\beta u(r)} e^{-\frac{\rho}{2} \int d \mathbf{r}^{\prime} \beta u\left(\mathbf{r}^{\prime}, \mathbf{r}\right)\left[h_{11}\left(r^{\prime}\right)-h_{12}\left(r^{\prime}\right)\right]}-1, \\
h_{12}(r)=e^{\beta u(r)} e^{\frac{\rho}{2} \int d \mathbf{r}^{\prime} \beta u\left(\mathbf{r}^{\prime}, \mathbf{r}\right)\left[h_{11}\left(r^{\prime}\right)-h_{12}\left(r^{\prime}\right)\right]}-1 .
\end{array}
$$

Finally, subtracting the two equations, we get a single relation

$$
h_{d}(r)=-2 \sinh \left[\beta u(r)+\frac{\rho}{2} \int d \mathbf{r}^{\prime} \beta u\left(\mathbf{r}-\mathbf{r}^{\prime}\right) h_{d}\left(r^{\prime}\right)\right] .
$$

Once the function $h_{d}(r)$ is obtained from the above selfconsistent relation, the pressure can be calculated from the virial equation

$$
\frac{\beta P}{\rho}=1-\frac{\pi \rho}{3} \int_{0}^{\infty} d r r^{3} \frac{\partial \beta u(r)}{\partial r} h_{d}(r) .
$$

In Fig. 11, we plot the resulting critical temperature isotherm at $T_{c}^{*} \approx 0.08$. This is slightly lower than that obtained from the RPA ( $T_{c}^{*} \approx 0.1$ in Fig. 6 ), yet not sufficiently close to the exact result $\left(T_{c}^{*} \approx 0.03\right.$ in Fig. 1$)$. The critical density within the present approximation is also slightly shifted, from $\rho_{c}^{*} \approx 0.06$ within the RPA to $\rho_{c}^{*} \approx 0.07$. In Fig. 12 , we plot the quantities $C_{i j}$, in analogy to Fig. 5 . The results show some improvement over the RPA, but still there is no indication of pair formation, as $C_{11}<0$ and $C_{12}<1$ for all parameters.

\section{Correlation functions from the GRPA approximation}

As in Sec. IV B, we define correlation functions as a perturbation of a uniform fluid caused by a fixed particle. In this section, however, we go beyond the mean-field level of description and use the generalized-RPA (GRPA), which is the RPA generalized to inhomogeneous fluids. ${ }^{15}$

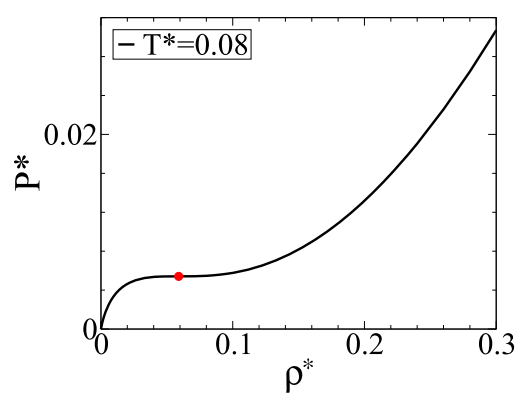

FIG. 11. Pressure isotherm at the critical temperature $T_{c}^{*} \approx 0.08$, obtained from Eqs. (25) and (26).

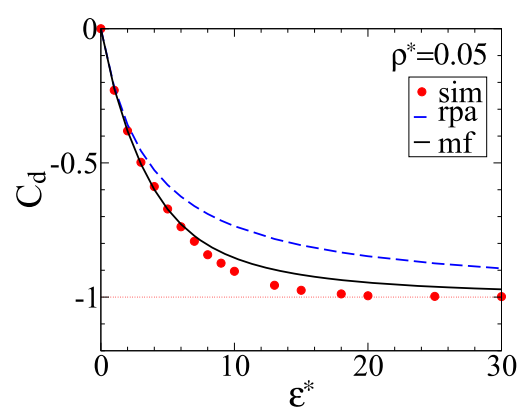

(a)

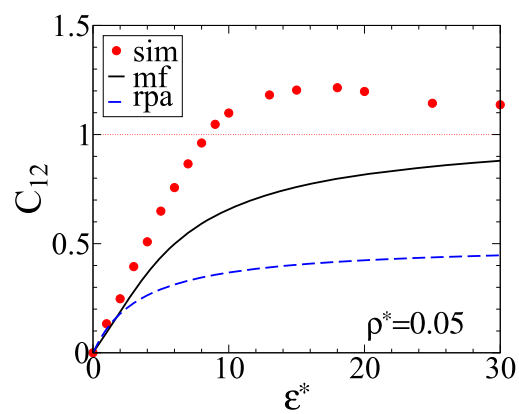

(b)

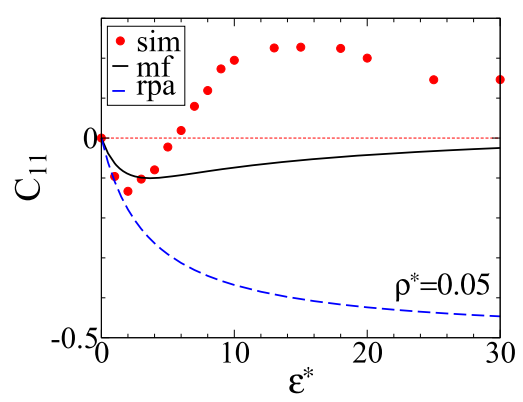

(c)

FIG. 12. The quantity $C_{i j}=\rho_{i} \int_{0}^{\infty} d r 4 \pi r^{2} h_{i j}(r)$ as a function of $\varepsilon^{*}$ for $\rho^{*}=0.05$.

Within the GRPA, the density perturbations caused by a fluid particle of the type " 1 " fixed at the coordinate origin are given by

$$
\begin{aligned}
& \rho_{1}(\mathbf{r})=\frac{\rho}{2} e^{-\beta u(r)} e^{-\beta w(r)} e^{\frac{1}{2}\left[H(\mathbf{r}, \mathbf{r})-H_{b}(0)\right]}, \\
& \rho_{2}(\mathbf{r})=\frac{\rho}{2} e^{\beta u(r)} e^{\beta w(r)} e^{\frac{1}{2}\left[H(\mathbf{r}, \mathbf{r})-H_{b}(0)\right]},
\end{aligned}
$$

with $w(\mathbf{r})$ defined in Eq. (22). The main difference between these expressions and those in Eqs. (20) and (21) is the presence of a correlation term $H(\mathbf{r}, \mathbf{r})$ obtained from

$H\left(\mathbf{r}, \mathbf{r}^{\prime}\right)=-\beta u(r)-\rho \int d \mathbf{r}^{\prime \prime}\left[h_{s}\left(r^{\prime \prime}\right)+1\right] \beta u\left(\mathbf{r}, \mathbf{r}^{\prime \prime}\right) H\left(\mathbf{r}^{\prime \prime}, \mathbf{r}^{\prime}\right)$,

which corresponds to the inhomogeneous Ornstein-Zernike equation with the direct correlation function approximated as $c\left(\mathbf{r}, \mathbf{r}^{\prime}\right)=-\beta u\left(\mathbf{r}, \mathbf{r}^{\prime}\right)$, and where $h_{s}(r)=\left[h_{11}(r)+h_{12}(r)\right] / 2$. The quantity $H_{b}(0)$ is the value of $H(\mathbf{r}, \mathbf{r})$ far away from a perturbation, where $\rho_{i}(r)=\frac{\rho}{2}$. Using the formal definitions $\rho_{1}(r)=\frac{\rho}{2}\left[h_{11}(r)+1\right]$ and $\rho_{2}(r)=\frac{\rho}{2}\left[h_{12}(r)+1\right]$, the correlation 


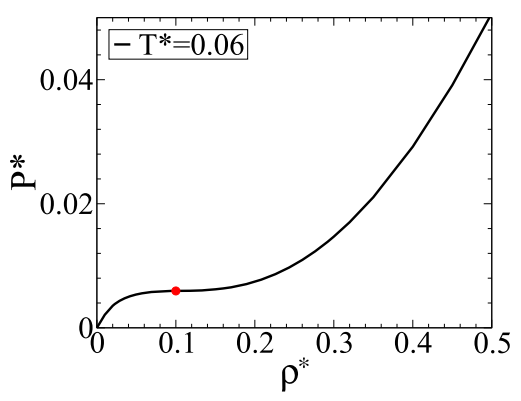

FIG. 13. Pressure isotherm at a critical temperature $T_{c}^{*}=0.06$ from the GRPA.

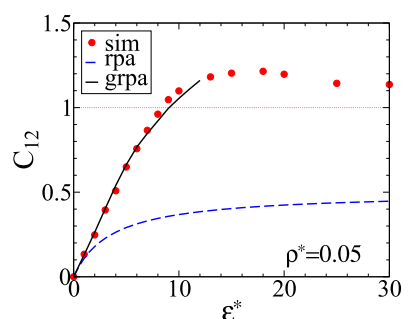

(a)

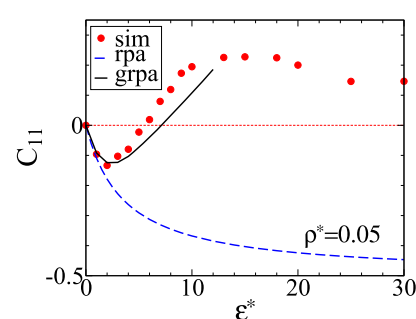

(b)
FIG. 14. The quantity $C_{i j}=\rho_{i} \int_{0}^{\infty} d r 4 \pi r^{2} h_{i j}(r)$ as a function of $\varepsilon^{*}$ for $\rho^{*}$ $=0.05$.

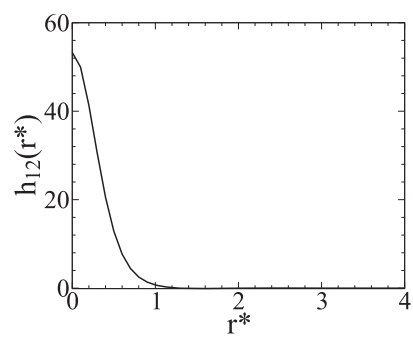

(a)

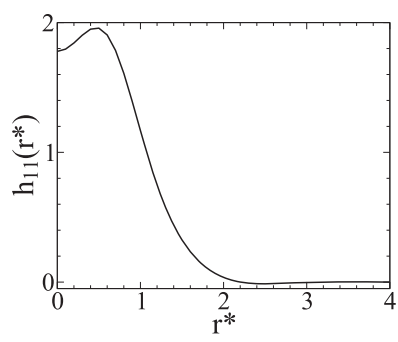

(b)
FIG. 15. Pair correlation functions from the GRPA, for $T^{*}=0.06$ and $\rho^{*}$ $=0.05$.

functions become

$$
h_{11}(r)=e^{-\beta u(r)-\frac{\rho}{2} \int d \mathbf{r}^{\prime} \beta u\left(\mathbf{r}-\mathbf{r}^{\prime}\right) h_{d}\left(r^{\prime}\right)} e^{\frac{1}{2}\left[H(\mathbf{r}, \mathbf{r})-H_{b}(0)\right]}
$$

and

$$
h_{12}(r)=e^{\beta u(r)+\frac{\rho}{2} \int d \mathbf{r}^{\prime} \beta u\left(\mathbf{r}-\mathbf{r}^{\prime}\right) h_{d}\left(r^{\prime}\right)} e^{\frac{1}{2}\left[H(\mathbf{r}, \mathbf{r})-H_{b}(0)\right]} .
$$

Once the correlation functions are calculated, we use Eq. (26) to calculate the pressure and to find the critical temperature isotherm.

The predicted critical temperature is $T_{c}^{*} \approx 0.06$, and the critical temperature isotherm is plotted in Fig. 13, where the critical density is $\rho_{c}^{*} \approx 0.1$. This, so far, is the best estimate, and at the same time, it is not accurate enough to be regarded as an accurate theory of the strong-coupling limit.

We look next into the quantities $C_{i j}$ shown in Fig. 14. A significant feature of the plots is the prediction of $C_{11}>0$, and $C_{12}>1$ for $\varepsilon^{*} \gtrsim 8$, indicating mediated attraction between particles of the same species and, therefore, the presence of dimers. The existence of dimers is further confirmed by the shape of the correlation function shown in Fig. 15 for low density and the temperature slightly above $T_{c}^{*}$. A sharp peak at $r=0$ for $h_{12}(r)$ indicates strong association between particles of the same species, and the absence of the correlation hole and the presence of positive correlations in $h_{11}(r)$ provide additional evidence for the existence of dimers within the GRPA.

\section{CONCLUSION}

A unique feature of the two-component system with the binary interactions of the form presented in Eq. (1) is the special role of correlations, which goes beyond a merely correctional role, and provides a mechanism for a gas-liquid phase-transition and the formation of dimers. Yet because correlations are dominant only in the strong-coupling limit, phase transition and the formation of dimers occur at very low temperatures. This, in consequence, makes theoretical analysis of these phenomena a challenging problem.

The simplest theory of correlations, the RPA, predicts the critical temperature at a significantly higher temperature than that obtained from simulations, and it fails to account for dimer formation. The most successful approximation attempted in this work is the GRPA. This approximation captures pair formation and yields the critical temperature that is closer to the simulation results, yet not close enough to be considered an accurate theory.

In consequence, a theoretical challenge of treating the two-component fluid with interactions in Eq. (1) remains open. An interesting direction to be considered is to study a relevant lattice-gas model, as was done for the one-component GCM fluid in Ref. 34.

Finally, based on our results, we do not find evidence that the existence of dimers plays a role in a phase transition mechanism. Pairs are prevalent at a low density, let us say $\rho^{*}<0.1$. The critical density, on the other hand, is at roughly $\rho_{c}^{*} \approx 0.6$. At such a high density, we no longer find any evidence for the existence of dimers, and so the link between pairs and the phase-transition is dubious. We regard the formation of dimers and the occurrence of the phase transition as two different manifestations of the strong-coupling limit. To provide support for this conjecture, we carried out simulations for permanent dimers (two Gaussian particles of different species connected by a spring). Such a contrived system exhibited no phase-transition. We conclude, therefore, that the interactions between particles at intermediate and high densities are considerably more complex than those provided by the simple reduction to dimers.

The prediction of dimers, however, can provide a useful test of a performance of an approximation. An approximation that predicts dimers can be assumed as appropriate for the strong-coupling limit and potentially suitable for accurate estimate of the critical point.

\section{ACKNOWLEDGMENTS}

This work was partially supported by the CAPES, CNPq, INCT-FCx, by the US-AFOSR under Grant No. FA9550-16-10280, and by PNDP-Capes under Project No. PNPD20132533. D.F. would like to acknowledge the usage of computational 
resources in the ESPCI ParisTech and the kind permission to do so by Tony Maggs and Michael Schindler.

${ }^{1}$ Y. Levin and M. E. Fisher, Phys. Rev. Lett. 71, 3826 (1993).

${ }^{2}$ Y. Levin and M. E. Fisher, Phys. A 225, 164 (1996).

${ }^{3}$ R. J. F. Leote de Carvalho and R. Evans, J. Phys.: Condens. Matter 8, 2245 (1996).

${ }^{4}$ A. Z. Panagiotopoulos, J. Phys.: Condens. Matter 17, S3205 (2005).

${ }^{5}$ A.-P. Hynninen and Z. Panagiotopoulos, Mol. Phys. 106, 2039 (2008).

${ }^{6}$ A. Nikoubashman, J.-P. Hansen, and G. Kahl, J. Chem. Phys. 137, 094905 (2012).

${ }^{7}$ D. Coslovich, J.-P. Hansen, and G. Kahl, Soft Matter 7, 1690 (2011).

${ }^{8}$ D. Coslovich, J.-P. Hansen, and G. Kahl, J. Chem. Phys. 134, 244514 (2011).

${ }^{9}$ D. Frydel and Y. Levin, J. Chem. Phys. 138, 174901 (2013).

${ }^{10}$ P. B. Warren, A. Vlasov, L. Anton, and A. J. Masters, J. Chem. Phys. 138, 204907 (2013).

${ }^{11}$ J.-M. Caillol and D. Levesque, J. Chem. Phys. 140, 214505 (2014).

${ }^{12}$ D. Frydel, J. Chem. Phys. 145, 184703 (2016).

${ }^{13}$ D. Frydel, Adv. Chem. Phys. 160, 209 (2016).

${ }^{14}$ Y. Levin, Rep. Prog. Phys. 65, 1577 (2002).

${ }^{15}$ D. Frydel and M. Man, Phys. Rev. E 93, 062112 (2016).

${ }^{16}$ Y. Xiang and D. Frydel, J. Chem. Phys. 146, 194901 (2017).
${ }^{17}$ W. Ebeling, Z. Phys. Chem. (Leipzig) 238, 400 (1968).

${ }^{18} \mathrm{H}$. Falkenhagen and W. Ebeling, in Ionic Interactions, edited by S. Petrucci (Academic Press, New York, 1971), Vol. 1.

${ }^{19}$ H. Watanabe, N. Ito, and C.-K. Hu, J. Chem. Phys. 136, 204102 (2012).

${ }^{20}$ F. H. Stillinger, J. Chem. Phys. 65, 3968 (1976).

${ }^{21}$ F. H. Stillinger and T. A. Weber, J. Chem. Phys. 68, 3837 (1978).

${ }^{22}$ F. H. Stillinger and T. A. Weber, Phys. Rev. B 22, 3790 (1980).

${ }^{23}$ F. H. Stillinger, J. Chem. Phys. 70, 4067 (1979).

${ }^{24}$ F. H. Stillinger, Phys. Rev. B 20, 299 (1979).

${ }^{25}$ F. H. Stillinger and D. K. Stillinger, Phys. A 244, 358 (1997).

${ }^{26}$ A. A. Louis, P. G. Bolhuis, and J. P. Hansen, Phys. Rev. E 62, 7961 (2000).

${ }^{27}$ A. J. Archer and R. Evans, Phys. Rev. E 64, 041501 (2001).

${ }^{28}$ B. Götzelmann, R. Roth, S. Dietrich, M. Dijkstra, and R. Evans, Europhys. Lett. 47, 398 (1999).

${ }^{29}$ R. Roth, R. Evans, and S. Dietrich, Phys. Rev. E 62, 5360 (2000).

${ }^{30}$ D. Goulding and S. Melchionna, Phys. Rev. E 64, 011403 (2001).

${ }^{31}$ F. H. Stillinger and R. Lovett, J. Chem. Phys. 48, 3858 (1968).

${ }^{32}$ F. H. Stillinger and R. Lovett, J. Chem. Phys. 49, 1991 (1968).

${ }^{33} \mathrm{Ph}$. A. Martin, Rev. Mod. Phys. 60, 1075 (1988).

${ }^{34}$ R. Finken, J.-P. Hansen, and A. A. Louis, J. Phys. A: Math. Gen. 37, 577 (2004). 